\title{
Multi-Perspective Decision-making Cloud Computing Adoption Model for Small and Medium Enterprises (SMEs)
}

\author{
Can Sayginer $^{1 *}$, Tuncay Ercan ${ }^{2}$ \\ ${ }^{1}$ Department of Management Information Systems, Yaşar University, University Street, No 37-39, Bornova, Izmir 35100, Turkey \\ ${ }^{2}$ Department of Computer Engineering, Yaşar University, University Street, No 37-39, Bornova, Izmir 35100, Turkey
}

\section{Abstract}

The research aims to test the model of integrated DOI theory and TOE framework to predict Cloud Computing (CC) decision-making intentions of SMEs, Izmir, Turkey. The survey data was collected from 140 Information Technology (IT) decision-makers via Google forms survey tool. Confirmatory factor analyses were made to examine the decision-making approach of SMEs. The study revealed that the variance of top management support and complexity explained $29.8 \%$ of the decision-making approach to CC adoption. The originality of the study is that the research on cloud computing in Turkey is scarce and not comprehensive either. In addition, they are not for Turkish SMEs. This research will bring together an introductory plan for cloud providers to understand the intentions of SMEs for adopting cloud computing. This research will also provide scholars with an in-depth analysis of the status contributing to the academic research in the field of ICT development in developing countries. This study will contribute to SMEs' ICT infrastructure policies, and support governments in creating a legal framework to make laws for a secure environment for SMEs to reduce costs, and gain a competitive advantage over Large Enterprises (LEs).

\section{Keywords:}

Cloud Computing Adoption;

Confirmatory Factor Analysis;

Decision-Making Model;

DOI Theory;

SMEs;

TOE Theory.

\section{Article History:}

Received: 25 August 2021

Revised: $\quad 17 \quad$ November 2021

Accepted: 03 December 2021

Published: 28 December 2021

\section{1- Introduction}

Information Technology (IT) has introduced several products and services such as software, internet, electronics, and telecommunication equipment that help business processes to be improved. IT also optimizes the costs budgets of the companies (economics perspective) [1]. From the social aspect, it creates a cooperative work environment [2]. It also increases individuals' knowledge so that they can comply with companies' operating processes (personal development) [3]. When these three perspectives are considered, the research question of this study is "what are the factors that affect, obstruct and threaten cloud technology adoption in small and medium enterprises". To have a better understanding and offer solutions to SMEs and cloud providers, the study analyzed the particular environment using Diffusion of Innovation (DOI) theory in combination with Technology, Organization and Environment (TOE) framework.

This study aims to provide a solution by showing the threats and hindrances to the perceptions of companies for Cloud Computing (CC) adoption in order to build the most effective and appropriate IT infrastructure system. This research also addresses the key challenges and issues of CC adoption, such as innovativeness of companies, technological, organizational, and environmental points of view. In order to build the rational mindset integrated with the business goals of companies in SMEs, this research fills the gap of literature for developing countries by

\footnotetext{
* CONTACT: can.sayginer@yasar.edu.tr

DOI: http://dx.doi.org/10.28991/esj-2021-SP1-010
}

(C) 2020 by the authors. Licensee ESJ, Italy. This is an open access article under the terms and conditions of the Creative Commons Attribution (CC-BY) license (https://creativecommons.org/licenses/by/4.0/). 
considering external factors, such as compatibility, competitive pressure, technological readiness, and top management support matters, and internal factors: cost reduction, security concerns, relative advantage, and complexity towards CC adoption. Besides the study of [4] about SMEs and the study of [5] about the logistic sector taking place in Turkey, there is no specific study applied DOI and TOE theories especially for Turkish SMEs in the field of cloud computing adoption.

CC offers sets of ICT infrastructure such as operating systems, backup services, and software for enterprises [6]. The deployment of ICT architecture consists of two business technology: on-premise, and CC [7]. The CC term, "data in the cloud", is declared as web-based computing with the idea of data, and software is hosted in the data centers of the providers to increase rented IT sources effectively and decrease operational expenditures (OPEX). However, businesses, which have highly sensitive data, fluctuating user demands, and diverse application alternation on a large number of users, tend to adopt on-premise computing that data and software are hosted in the local servers [7]. Either of the decision varied different challenges for companies but most companies faced with the issues of analyzing, implementing IT activities for their business processes that cause high variable costs [8]. Therefore, understanding the difference between on-premise and CC is important for the IT infrastructure decisions of companies to form business continuity. There are several IT infra-structure studies for SMEs regarding CC adoption. Keskin et al. (2020) [9] observed the critical elements of cloud provider selection over storing data (on-premise or CC). Neicu et al. (2020) [10] analyzed the perception of cloud consumers over using cloud providers' product. Nagahawatta et al. (2021) [11] made an in-depth literature research from 2010 to 2020 regarding security concerns over CC adoption. Hence, the scholars found out that security is important as knowing data, where should be located, is important either outside of company or inside, and knowing the customers' ability to learn CC products is also crucial to overcome productivity issues.

Most companies, which adopted cloud computing (CC), generate "meaningful data" for companies to sustain their existence in the market [12]. Due to the challenges faced and difficulties of storing and analyzing a large amount of data in companies, CC has become a breakthrough technology for the last decade. There is no doubt that this inevitable technology adoption tends to increase because of ever-increasing IP traffic growth, broadband speed, and mobile traffic growth. There is tremendous growth in the number of data traffic at the rate of $22 \%$, the broadband rate at nearly $100 \%$, mobile traffic at the rate of 53\% from 2015 to 2020 [13]. Cisco (2020) [14] also reported that data traffic, broadband rate, and mobile traffic are expected to increase up to 0.35 -fold, 4.2 -fold, and 0.57 -fold growth by 2023 , respectively. Hence, this ever-increasing volume of internet users with the data growth indicated that examining the benefits, challenges, and obstacles of cloud computing adoption cannot be ignored. In other words, this study is necessary since companies ought to take affordable IT solutions according to their business needs against everincreasing data growth in the world, and position themselves for the market competition to smoothly utilize the benefits of cloud computing adoption.

Turkey is the $17^{\text {th }}$ largest economy with a GDP of 857 billion USD [15]. Sener et al. (2014) [4] also stated that SMEs constitute $99 \%$ of the total number of companies and cover $76 \%$ of the employment of the Turkish industry. Although a great percentage of the number of SMEs are small and they employ up to 675800 workers, which can be considered as companies with low potential for cloud computing, the medium-size enterprises that employ 1.275 .772 workers each employing up to 250 employees form a great potential for cloud adoption [4]. SMEs are also the innovator promoters in developing countries [16].

According to OC \& C Strategy Consultants (2018) [15], CC services have increased the level of innovativeness of a country. Turkey's fiber broadband services have increased $10 \%$ percent from 2016 to 2018 but should increase more to attract cloud providers for entering the Turkish ICT market. Free cross-border data flows have caused the accessibility of cloud-based services of Infrastructure as a service (IaaS), Software as a service (SaaS), and Platform as a service (PaaS) to grow in Turkey [15]. Hence, Deloitte (2020)'s research [17] also revealed that CC will be highly impactful in technological areas in Turkey in the next years.

\section{2- Theoretical Framework}

The issues of CC adoption emerged and were addressed in some technological decision theories. These adoption theories are widely used for assessing factors of CC adoption for example, Diffusion of Innovation (DOI) (Rogers, 1983) [18]; Technology, Organization and Environment (TOE) (Tornatzky and Fleischer, 1990) [19]; Technology Acceptance Theory (TAM) (Davis, 1989) [20]; and The Theory of Reasoned Action (TRA) (Fishbein and Ajzen, 1977) [21]. In this study, Integrated DOI and TOE theory models were applied for Turkish SMEs, as illustrated in Figure 1. Due to the need for organizational research, combined DOI and TOE theory is considered effective to use, as it is the two-step flow decision (adoption or rejection) of DOI theory, mentioned by Rogers (1983) [18], TOE theory was incorporated into this study together with DOI theory to exhibit how external environment of CC adoption affects the decision processes of SMEs. There are two adoption theories used for assessing factors of cloud computing adoption in the study. 


\section{2-1-Diffusion of Innovation (DOI) Theory}

Relative advantage is the degree, which understands the perceived manner of individuals over the new technology in terms of economic factors, and social-prestige factors, over the old technology [22]. Security concerns and cost reduction are found as economic and social-prestige factors by Oliveira et al. (2014) [23]. Complexity is the degree, to understand the perceived behavior of individuals that are found easy or difficult to use [22]. In Turkish SMEs, Cost Reduction (CR), Security Concerns (SC), and Relative Advantage (RA) indirectly affected CC adoption but complexity affected CC adoption directly, as the hypothesis shown below.

H1a1: Cost Reduction (CR) will negatively influence relative advantage (RA) for SMEs.

H1a2: Security Concerns (SC) will negatively influence relative advantage (RA) for SMEs.

H1a: Relative Advantage (RA) will negatively influence complexity (CX) for SMEs.

H1: Complexity (CX) will negatively influence cloud-computing adoption (CCA) for SMEs.

\section{2-2-Technology, Organization, and Environment (TOE) Theory}

Top management leadership behavior is an organizational context, which has a key role in fostering CC adoption. The executive team is formed by top management support for the adoption to create a vision for the future [19]. Compatibility is the degree, to which one understands the perceived ideas, norms, and also whether they fit the previous experiences and the existing values of individuals [22]. The available technology is a technological context, in which the company's resources are supposed to fit with the current technology. Technological readiness was pioneered by Tornatzky \& Fleischer (1990) [19] to understand the constraints and capacity of companies over the technology choice. The dimension of competition is an environmental context, in which the price, quality, and service give companies the ability to compete with the rivals [19]. Competitive pressure was found by Tornatzky \& Fleischer (1990) [19] to monitor the differences in communication with customers. In Turkish SMEs, Technological Readiness (TR), Compatibility (CO), and Competitive Pressure (CP). Top management support directly affected CC adoption, as the hypothesis illustrated below.

H2: Top Management Support (TMS) will positively influence cloud-computing adoption (CCA) for SMEs.

H2a: Compatibility (CO) will positively influence Top Management Support (TMS) for SMEs.

H2b: Technological Readiness (TR) will positively influence Top Management Support (TMS) for SMEs.

H2c: Competitive Pressure (CP) will positively influence Top Management Support (TMS) for SMEs.

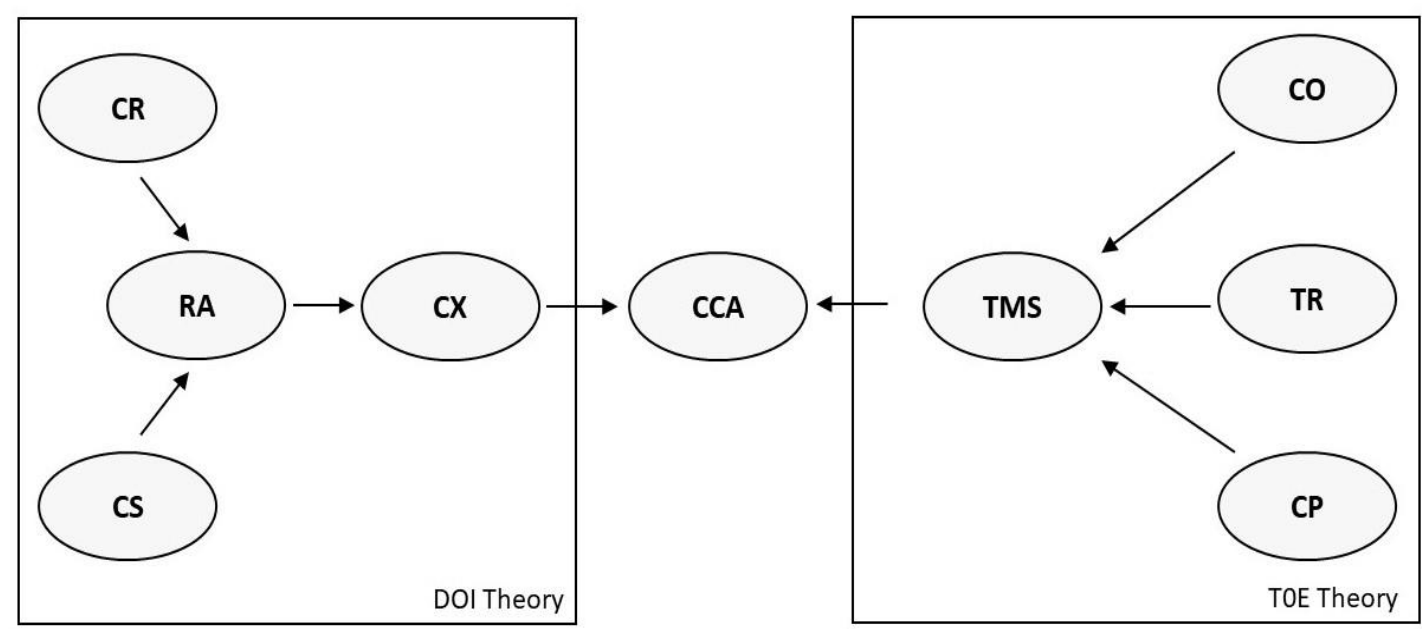

Figure 1. The proposed model.

\section{3- Research Methodology}

The research strategy is mainly survey research that contains questionnaires as the data collection method. The quantitative research methodology was chosen for SMEs to investigate innovation diffusion (DOI theory) and the technology, organization, and environment factors of CC adoption (TOE theory). The survey was sent to 472 SMEs from the Izmir Chamber of Commerce, and the Aegean Region Chamber of Industry. The survey was responded to by 140 SMEs (the response rate is 29.6\%) that fits the sample size, which should be 3 to 20 times the number of variables and range from 100 to above 1000 according to Mundform et al. (2005) [24]. Accordingly, Confirmatory factor analysis was used to analyze the data over the SmartPLS analysis tool. The research methodology flow chart was illustrated in Figure 2 below. 


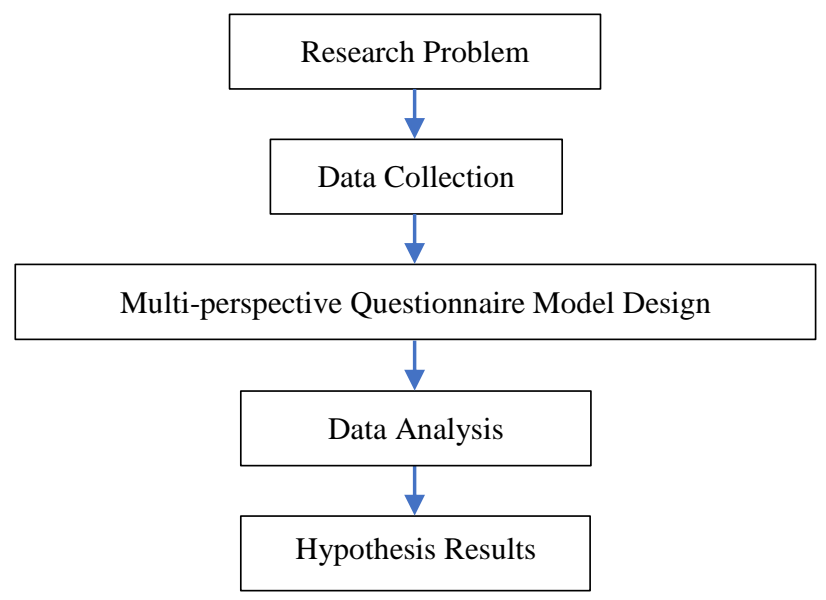

Figure 2. The Research Methodology Flow Chart.

The unit of analysis is the SMEs, classified as previously experienced CC adoption, already adopted, or never adopted CC in the city of Izmir, Turkey. Google forms were used for online questionnaires. 32 close-ended questions were asked to respondents from January 2021 to June 2021. Out of 32, questions 6 were created to profile SMEs' IT capacities, as shown in Table 1. 26 questions were gathered from Oliveira et al. (2014)'s study [23]. The proposed model is not completely consistent with Oliveira et al. (2014)'s research model [23]. There are differences because this study was conducted in a different country with a unique business and industrial climate. Tornatzky \& Klein (1982) [25] declared that complexity and compatibility both have relationships with DOI and TOE theories, in which compatibility is included for the proposed model in the TOE model.

The Likert scale (strongly disagree 1-5 strongly agree) was used to gather ratio scale data. In the proposed model 9 constructs and 26 items were measured by applying structural equational modeling. CC adoption was selected as a dependent variable containing 2 items. In the DOI theory, there are 4 independent variables including RA, CR, CS, and CX each of which has 4, 2, 3, and 3 items, respectively. In the TOE theory, there are 4 independent variables including CO, TR, CP, and TMS that each of them has 4, 2, 3, and 3 items, respectively. Upon eliciting the results, evaluations were made based on the hypothesis suggested as a starting point for the study.

\section{4- Results}

Table 1 indicated that $44.2 \%$ of SMEs in Turkey have not adopted CC yet. Within $44.2 \%, 12.1 \%$ of Turkish SMEs are expected to adopt CC in the next 3 years or more. The percentages of those SMEs which are expected to adopt CC in less than a year, between 1 and 3 years, and 3 years and above are all the same as $10.7 \%$. The project managers, who agreed to implement or decided not to implement CC, were male (67.1\%), between the age of 41 and 50 (33.6\%), and their SMEs' operating in the service sector (77.8\%). Cloud applications, which have been adopted by SMEs, are IaaS (Cloud Storage, and Virtual Servers), PaaS (Microsoft Azure, Google App Engine, Amazon EC2 cloud platforms, and IBM SmartCloud Cloud-based Database Management Systems. Amazon RDS, Google Cloud SQL, Microsoft SQL Azure, etc.), and SaaS (Google apps, CRM, ERP, etc.) were with 53.5\%, 21.4\%, and 18.5\%, respectively.

Table 1. Small and medium enterprises (SMEs) profile of the study.

\begin{tabular}{clcc}
\hline & SMEs Profile & & \\
\hline \multirow{2}{*}{ Gender } & & Number & $(\%)$ \\
\hline \multirow{2}{*}{ Age } & Male & 94 & 67.1 \\
& Female & 46 & 32.9 \\
\hline \multirow{2}{*}{ Sector } & $20-25$ & 9 & 6.43 \\
& $26-30$ & 30 & 21.4 \\
& $31-40$ & 47 & 33.5 \\
& $41-50$ & 30 & 21.4 \\
& 51 and above & 24 & 17.1 \\
\hline \multirow{3}{*}{ Number of Cloud Applications } & Production Sector & 31 & 22.2 \\
& Service Sector & 109 & 77.8 \\
\hline & 0 & 62 & 44.2 \\
& Between 1 and 3 & 53 & 37.8 \\
& Between 4 and 6 & 16 & 11.4 \\
& 7 and above & 9 & 8.2 \\
\hline
\end{tabular}




\begin{tabular}{clcc}
\hline & Companies don't consider it & 15 & 10.7 \\
& Less than 1 year & 15 & 10.7 \\
The Expected time for the adoption & Between 1 and 3 years & 15 & 10.7 \\
& 3 years and above & 17 & 12.1 \\
& Companies already adopted it & 78 & 57.4 \\
\hline \multirow{2}{*}{ Cloud Applications } & Infrastructure services & 75 & 53.5 \\
& Platform services & 30 & 21.4 \\
& Software services & 26 & 18.5 \\
& Security as a Service, etc. & 9 & 6.4 \\
& Non adopters & 78 & 44.2 \\
\hline
\end{tabular}

DOI and TOE constructs demonstrate composite reliability (CR), and average variance extracted (AVE) that are between 0.841, and 0.929, which are between above 0.7, and 0.5 based on Arifin (2019) [26], respectively. It shows reliable and valid data, as shown in Table 2. For the integrated DOI and TOE theory, all items' loadings are greater than 0.7 declared by Hair et al. (2010) [27], which is valid. Thus, all items are chosen in the structural model.

DOI has four constructs. For relative advantage, four items were added, containing the value of CR (0.912), including the value of AVE (0.721), involving the value of Cronbach's Alpha (0.870), and holding factor loadings between 0.591 and 0.888 . For cost reduction, two items were added, containing the value of CR (0.842), including the value of AVE (0.728), involving the value of Cronbach's Alpha (0.632), and holding factor loadings between 0.808 and 0.896 . For security concerns, three items were added, containing the value of CR (0.921), including the value of AVE (0.796), involving the value of Cronbach's Alpha (0.875), and holding factor loadings between 0.859 and 0.910 . For complexity, three items were added, containing the value of CR (0.912), including the value of AVE (0.776), involving the value of Cronbach's Alpha (0.855), and holding factor loadings between 0.783 and 0.942 .

TOE has four constructs. For compatibility, four items were added, containing the value of CR (0.929), including the value of AVE (0.765), involving the value of Cronbach's Alpha (0.898), and holding factor loadings between 0.752 and 0.897 . For technological readiness, two items were added, containing the value of CR (0.844), including the value of AVE (0.730), involving the value of Cronbach's Alpha (0.636), and holding factor loadings between 0.839 and 0.870 . For competitive pressure, three items were added, containing the value of CR (0.841), including the value of AVE (0.640), involving the value of Cronbach's Alpha (0.728), and holding factor loadings between 0.731 and 0.900. For top management support, three items were added, containing the value of CR (0.891), including the value of AVE (0.733), involving the value of Cronbach's Alpha (0.817), and holding factor loadings between 0.747 and 0.910 .

The independent construct is cloud computing adoption. For cloud computing adoption, two items were added, containing the value of CR (0.967), including the value of AVE (0.937), involving the value of Cronbach's Alpha (0.933), and holding factor loadings between 0.966 and 0.970 .

According to Table 4, the structural model fits well with the SRMR value of 0.078, which Hu \& Bentler (1998) [28] confirmed that SRMR is less than 0.08 , or 0.10 , a good fit, or average fit, respectively.

Table 2. DOI and TOE constructs, items, loadings, Cronbach's Alpha, CR, and AVE values.

\begin{tabular}{|c|c|c|c|c|c|}
\hline DOI Main Constructs & Items & Loadings & Cronbach's Alpha & CR & AVE \\
\hline \multirow{4}{*}{ (H1a) Relative Advantage (RA) } & RA1 & 0.888 & \multirow{4}{*}{0.870} & \multirow{4}{*}{0.912} & \multirow{4}{*}{0.721} \\
\hline & RA2 & 0.880 & & & \\
\hline & RA3 & 0.822 & & & \\
\hline & RA4 & 0.591 & & & \\
\hline \multirow{2}{*}{ (H1a1) Cost Reduction (CR) } & CR1 & 0.896 & \multirow{2}{*}{0.632} & \multirow{2}{*}{0.842} & \multirow{2}{*}{0.728} \\
\hline & CR2 & 0.808 & & & \\
\hline \multirow{3}{*}{ (H1a2) Security Concerns (SC) } & SC1 & 0.910 & \multirow{3}{*}{0.875} & \multirow{3}{*}{0.921} & \multirow{3}{*}{0.796} \\
\hline & SC2 & 0.859 & & & \\
\hline & SC3 & 0.908 & & & \\
\hline \multirow{3}{*}{ (H1) Complexity (CX) } & CX1 & 0.783 & \multirow{3}{*}{0.855} & \multirow{3}{*}{0.912} & \multirow{3}{*}{0.776} \\
\hline & CX2 & 0.942 & & & \\
\hline & CX3 & 0.911 & & & \\
\hline
\end{tabular}




\begin{tabular}{|c|c|c|c|c|c|}
\hline TOE Main Constructs & Items & Loadings & Cronbach's Alpha & $\mathbf{C R}$ & AVE \\
\hline \multirow{4}{*}{ (H2a) Compatibility (CO) } & CO1 & 0.897 & \multirow{4}{*}{0.898} & \multirow{4}{*}{0.929} & \multirow{4}{*}{0.765} \\
\hline & $\mathrm{CO2}$ & 0.873 & & & \\
\hline & $\mathrm{CO3}$ & 0.877 & & & \\
\hline & $\mathrm{CO4}$ & 0.752 & & & \\
\hline \multirow{2}{*}{ (H2b) Technological Readiness (TR) } & TR1 & 0.839 & \multirow{2}{*}{0.636} & \multirow{2}{*}{0.844} & \multirow{2}{*}{0.730} \\
\hline & TR2 & 0.870 & & & \\
\hline \multirow{3}{*}{ (H2c) Competitive Pressure (CP) } & CP1 & 0.900 & \multirow{3}{*}{0.728} & \multirow{3}{*}{0.841} & \multirow{3}{*}{0.640} \\
\hline & CP2 & 0.731 & & & \\
\hline & CP3 & 0.759 & & & \\
\hline \multirow{3}{*}{ (H2) Top Management Support (TMS) } & TMS1 & 0.910 & \multirow{3}{*}{0.817} & \multirow{3}{*}{0.891} & \multirow{3}{*}{0.733} \\
\hline & TMS2 & 0.747 & & & \\
\hline & TMS3 & 0.809 & & & \\
\hline Independent Construct & Items & Loadings & Cronbach's Alpha & $\mathbf{C R}$ & AVE \\
\hline \multirow{2}{*}{ Cloud Computing Adoption (CCA) } & CCA1 & 0.966 & \multirow{2}{*}{0.933} & \multirow{2}{*}{0.967} & \multirow{2}{*}{0.937} \\
\hline & CCA2 & 0.970 & & & \\
\hline
\end{tabular}

The correlation matrix was measured within 9 constructs. According to the three dimensions of correlations pointed by Hair et al. (2017) [29], the strongest correlations were listed in Table 3, below. It was observed that there is a strong correlation between compatibility, and top management support, with a value of 0.734 . It was found that there is a partial correlation between technological readiness, and top management support, with a value of 0.679 . It can be stated that there is a partial correlation between compatibility, and technological readiness, with a value of 0.629 .

Table 3. Correlation matrix.

\begin{tabular}{|c|c|c|c|c|c|c|c|c|c|}
\hline Constructs & $\mathbf{R A}$ & CR & SC & CX & $\mathrm{CO}$ & TR & $\mathbf{C P}$ & TMS & CCA \\
\hline RA & 1 & & & & & & & & \\
\hline $\mathrm{CR}$ & 0.528 & 1 & & & & & & & \\
\hline $\mathrm{SC}$ & -0.168 & 0.088 & 1 & & & & & & \\
\hline $\mathrm{CX}$ & -0.399 & -0.200 & 0.494 & 1 & & & & & \\
\hline $\mathrm{CO}$ & 0.626 & 0.362 & -0.250 & -0.332 & 1 & & & & \\
\hline TR & 0.427 & 0.223 & -0.091 & -0.268 & 0.629 & 1 & & & \\
\hline $\mathrm{CP}$ & 0.343 & 0.224 & -0.620 & 0.046 & 0.452 & 0.332 & 1 & & \\
\hline TMS & 0.588 & 0.319 & -0.168 & -0.271 & 0.734 & 0.679 & 0.331 & 1 & \\
\hline $\mathrm{CCA}$ & 0.404 & 0.162 & -0.321 & -0.295 & 0.507 & 0.282 & 0.331 & 0.522 & 1 \\
\hline
\end{tabular}

*Three dimensions of correlations (Hair et al., 2017) [25]: below 0.3: weak, 0.3-0.7: partial, above 0.7: strong

The relationship between the constructs was referred to by the hypothesis testing, as shown in Table 4 . T values of the constructs, which are higher than 1.96, or less than -1.96 , verifying that the hypothesis is supported [30].

For DOI theory, the study exhibited that CR positively affects RA with a strong effect size $(\beta=0.547, p=0.000)$, with a $\mathrm{T}$ value of 7.748 , thus supporting H1al but SC negatively affects RA with a strong effect size $(\beta=-0.216$, $\mathrm{p}=0.011$ ), with $\mathrm{T}$ value of -2.561 thus supporting H1a2. The study showed that RA negatively affects CX with a strong effect size $(\beta=-0.399, \mathrm{p}=0.000)$, with the $\mathrm{T}$ value of -4.822 respectively, thus supporting $\mathrm{H} 1 \mathrm{a}$. The study indicated that $\mathrm{CX}$ negatively affects $\mathrm{CCA}$ with a strong effect size $(\beta=-0.166, \mathrm{p}=0.020)$, with the $\mathrm{T}$ value of -2.337 respectively, supporting $\mathrm{H} 1$.

For TOE theory, the study indicated that CO positively affects TMS with a strong effect size $(\beta=0.398, p=0.00)$, with the $\mathrm{T}$ value of 4.906 respectively, thus supporting H2a. The study exhibited that TR positively affects TMS with a strong effect size $(\beta=0.340, p=0.000)$, with the $\mathrm{T}$ value of 4.800 , thus supporting $\mathrm{H} 2 \mathrm{~b}$. The study showed that $\mathrm{CP}$ positively affects TMS with a strong effect size $(\beta=0.269, \mathrm{p}=0.000)$, with the T value of 4.424 , thus supporting H2c. The study exhibited that TMS positively affects CCA with a strong effect size $(\beta=0.477, \mathrm{p}=0.000)$, with the T value of 7.189, thus supporting $\mathrm{H} 2$. 
The variances of the integrated model are seen in Table 4. The variance of CR, and SC explained $32.5 \%$ of the variance of RA. The variance of RA explained $15.9 \%$ of the variance of CX. The variance of CO, TR, and CP explained $67.4 \%$ of the variance of TMS. The variance of CX, and TMS explained $29.8 \%$ of the variance of RA. The results indicate that SMEs with decisions of top management support, and the degree of complexity highly influence $\mathrm{CC}$ adoption. The path diagram of the proposed model is shown in Figure 3.

Table 4. Results of Hypothesis testing.

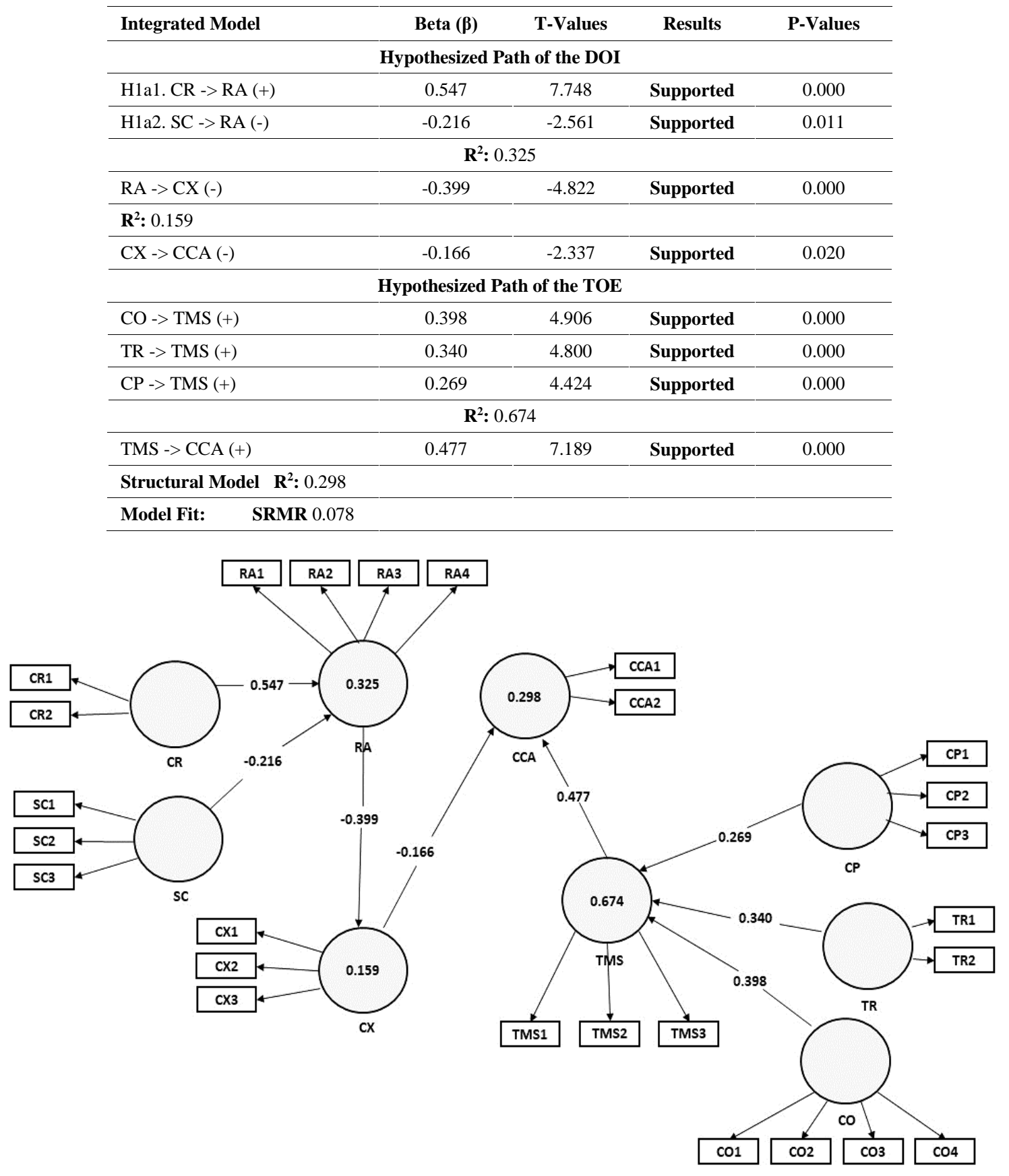

Figure 3. The path diagram of the proposed model.

\section{5- Discussions}

\section{5-1-Main Findings of the Present Study}

$\mathrm{CC}$ is not widespread in Turkish SMEs and is not expected to be widely used soon, as illustrated in Table 1 . The DOI theory, which measured how it is spread in the Turkish SMEs market, showed that the degree of complexity both negatively and directly influences $\mathrm{CC}$ adoption. Investigating the external factors, the TOE theory, indicated that the top management support directly influenced the decision-making approach to adopt CC for Turkish SMEs. Both of the factors showed that business processes adoption, and steering committee and their management took an important role in the decision. 


\section{5-2-Implication and Explanation of Findings and Comparison with Other Studies}

One of the most effective factors of complexity is cost savings. Fixed costs should be specified as the cost of ownership in terms of equipment costs, data migration costs, and maintenance costs [31]. If variable costs are a large amount, vanilla implementation can be applied by the cloud provider as the default system. If a company is eager to customize it, if required, hiring a consultant can diminish the costs of ownership that SMEs can know what their needs are, which can result in the reduction of a degree of complexity [7]. Al Ajmi et al. (2017), Bhuyan \& Dash (2018), and Lynn et al. (2018) [32-34] stated that cost reduction affected CC adoption in the education sector in Umman, Indian hospitals, and Irish companies. However, Oliveira et al. (2014) [23] disagreed that cost reduction didn't directly affect $\mathrm{CC}$ adoption in service and production companies in Portugal. Cost-saving is more likely to affect SMEs Alismaili et al. (2016) and it also affects companies that use SaaS Janssen \& Joha $(2011)$ [35, 36].

The second of the most effective factors of complexity is security concerns. Maintenance, network configurations, software license, and log-in servers are set by cloud providers, instead of SME's own IT department. This has brought several risks such as eavesdropping, denial of service attacks that have brought complexity in configuring and customizing cloud services. Disaster recovery can be applied to cloud-based services, which has brought certainty in adopting CC. On the other hand, Charlebois et al. (2016) [37] appealed that the security concerns will negatively influence relative advantage in genomics research in Germany, Lynn et al. (2018) [34] stated that the security concerns will negatively influence relative advantage in Irish companies, Sayginer \&Tuncay (2020) [38] reported that the security concerns will negatively influence relative advantage in Turkish national sector. Security concerns are more likely to be supported in developed countries, based on Charlebois et al. (2016) [37], and Lynn et al. (2018) [34].

The third most effective factor of complexity is the relative advantage. $\mathrm{CC}$, the new system, has brought a set of initiatives such as hiring technical equipment, renting software based on the fluctuating number of varying users and over the legacy system. This has made the implementation process faster, and it enables SMEs to introduce their products and services to the market much quicker. Alshamaila et al. (2013), and Sallehudin et al. (2015) [39, 40] agreed that relative advantage directly affects CC adoption in English SMEs, and the Malaysian public sector, respectively. However, Charlebois et al. (2016), and Hassan and Nasir (2017) [37, 41] contradicted that relative advantage will not directly influence CC adoption. Relative advantage is less likely to be affected SMEs in the Far East (Hassan and Nasir, 2017), the middle eastern firms (Mas'adeh, 2016), and in the colleges, and universities of the American continent (Klug \& Bai, 2015) [41-43]. Relative advantage is more likely to affect European SMEs' decisions (Alshamaila et al., 2013), and in developing countries (Senyo et al., 2016) [39, 44].

One of the most effective factors of CC adoption is complexity. If the business processes adoption issues emerge, bolt-in technology can be offered to build much-customized software from a third-party cloud provider [7]. Adopting multiple products from distinct cloud providers has brought the lack of building centralized database systems. In case of this breakdown, a best-of-breed strategy can be recommended to adopt multiple products from several cloud providers, but SLA contracts should be done in case of the exit strategy. Aktepe \& Saatcioglu (2017) [5] stated that the application properties, which cloud providers have built, are restricted for logistic sectors. Sallehudin et al. (2015), and Hassan and Nasir (2017) [40, 41] claimed that the complexity doesn't affect CC adoption in the Malaysian public sector and Malaysian SMEs, respectively. However, Oliveira et al. (2014), Gangwar et al. (2015), Gutierrez et al. (2015), and Alkhalil et al. (2017) [23, 45-47] found the complexity is significantly important in the adoption in Portuguese companies, Indian companies, English companies, and English SMEs, respectively. Complexity is a widely accepted factor of DOI and TOE in the research overviewed prior to this study such as Oliveira et al. (2014), Gangwar et al. (2015), Gutierrez et al. (2015), Alkhalil et al. (2017), and Alshamaila et al. (2013) [23, 39, 45-47], whereas public sector is not commonly accepted.

The second of the most effective factors of CC adoption is top management support. A steering committee of SMEs in Turkey should appoint an experienced project manager. Consultants can take part in the system to customize cloud-based services. Parallel implementation can also be applied, as the old system is in progress, the new system can work at the same time as well. A project manager should hire qualified program managers to test units, integrations, and performance loads. If the business process is already fit, a big bang implementation strategy can be applied, which decreases the cost. Oliveira et al. (2014) [23] found that top management support highly affects the adoption in the Portuguese service sector and companies; Alkhalil et al. (2017) [47] reported that it highly affects the adoption in English SMEs; Deil \& Brune (2017) [48] stated that top management support highly affects the adoption German SMEs. However, Oliveira et al. (2014), Gutierrez et al. (2015), Kyriakou et al. (2017), and Al-Hujran et al. (2018) [23, $46,49,50]$ proved that top management support is not significantly important, in the Portuguese manufacturing sector, UK companies, ceramic and cement sectors in six European countries (Germany, France, Italy, Poland, Spain, and the UK) and Jordan companies. Top management support is more likely to affect the service sector declared by Oliveira et al. (2014) and SMEs stated by Alkhalil et al. (2017) [23, 47].

The first of the most effective factors of top management support is compatibility. Well-defined roles and targeted departmental communications should be specified to increase knowledge of CC in Turkish SMEs. Super-users can be 
deployed in the project to build strong communication skills among the staff [7]. In case of any failures, change agents can be hired to help minimize compatibility issues in the Turkish market with sets of educations for staff to use the new system (Bradford, 2015) [7]. Otherwise, these actors can result in losing the power of top management support in the future for Turkish SMEs. Deil \& Brune (2017), Bhuyan \& Dash (2018), and Lynn et al. (2018) [48, 33, 34] proved that compatibility affects CC adoption in German SMEs, Indian hospitals, Irish SMEs, respectively. On the other hand, Oliveira et al. (2014), Alismaili et al. (2016), and Hassan and Nasir (2017) [23, 35, 41] claim that perceived compatibility is not likely to affect adoption in Portuguese companies, Australian SMEs, and Malaysian SMEs, respectively. Compatibility is claimed to be more likely to affect English SMEs Alshamaila et al. (2013) [39], it is also more likely to affect SMEs in Asia Continent Pathan et al. (2017) [51], Senyo et al. (2016) [44] reported that compatibility is likely to affect developing countries; Sallehudin et al. (2015) [40] found out that it is highly effective in far east public sector; Mas'adeh (2016) [42] reported similar likeliness in Middle Eastern countries. Studies in Middle Eastern higher education institutions and Tashkandi and Al-Jabri (2015) [52] shoved similarities.

The second of the most effective factors of top management support is technological readiness. The SMEs, which use internet infrastructure, and mobile technology, should consider technological readiness [46]. A high-bandwidth connection is essential for providing quality of service that the availability and the response times of services must be guaranteed under the SLA [31]. Adequate broadband access in Turkey hasn't been made according to the user needs $[53,54]$. There is no national broadband plan strategy implemented [55]. However, The Scientific and Technological Research Council of Turkey (TUBITAK), the Turkish GSM operators such as Turk Telekom, and Turkcell started investing in base stations for 5G internet infrastructure [56]. Oliveira et al. (2013), Oliveira et al. (2014), and Hassan et al. (2017) $[23,57,58]$ asserted that technological readiness was found significantly important, regarding Portuguese companies, Portuguese firms, and Malaysian SMEs, respectively, however, Alkhalil et al. (2017), and Deil and Brune (2017) [47, 48] opposed the perceived technological readiness effects on CC adoption in English SMEs and German SMEs. Technological readiness is less likely to affect small and medium companies in developed countries [23, 47, 48].

The third of the most effective factors of top management support is competitive pressure. Competitive pressure over companies depends on the SaaS, PaaS, and IaaS cloud provider's products and entry-level of Turkish market that affects top management of companies to build an IT infrastructure strategy. The bargaining power of buyers of infrastructure as a service is weak in Turkey over PaaS, and SaaS, as there are fewer IaaS cloud providers such as Amazon, Google, and DigitalOcean [59]. As indicated in Table 1, there are 34.4\%, 13.8\%, and 11.9\% of IaaS, PaaS, and SaaS adopters for Turkish SMEs, respectively. The majority of SMEs have adopted IaaS, which shows that wellknown cloud providers have absolute power over adopting CC. On the other hand, PaaS, and SaaS adopters are less likely to adopt since switching cost is less and the exit barrier is less difficult than IaaS. Gangwar et al. (2015), and Hassan et al. (2017) $[45,58]$ reported that there is a significant relationship between competitive pressure and the adoption in Indian companies and Malaysian SMEs. On the other hand, Oliveira et al. (2014), and Alismaili et al. (2016) $[23,35]$ claimed that competitive pressure is not significantly important. Competitive pressure has changed depending on the region $[45,58]$. Far East countries are also more likely to be affected in Indian and Malaysian companies Gangwar et al. (2015) [45, 58] respectively.

\section{5-3-Strengths and Limitations}

This study will be useful for SMEs to increase the level of the adoption at a high compliance level for ICT development in terms of IaaS, PaaS, and SaaS adoption to compete with LEs. This study contributes to IT development through cloud providers and academia by applying integrated DOI and TOE theory on how SMEs can better understand their business processes and how to deal with the drawbacks and barriers of adopting CC for the decision-making approach to CC adoption. This study will help non-cloud adopters increase their knowledge of CC in terms of the development of ICT infrastructures for the successful digital transformation. The main restriction of the study is that the study has been conducted only in a specific region of Turkey, however, the study topic, CC adoption, is considered a common issue in developing countries with similar businesses and industrial climate.

\section{6- Conclusion, Recommendation, and Future Work}

There is a low level of CC adoption at a low compliance level by Turkish SMEs, as it is shown that adopters are more likely to adopt IaaS than PaaS and SaaS. There is also a low-security level as it is seen in Table 1 that 4 out of 100 SMEs there is security as service users (SeaaS) only, as it is also believed that the remote data centers from the office are untrustworthy. Business process adoption issues in terms of complexity, and inexperienced project managers in terms of top management support are the key hindrances on the CC adoption for Turkish SMEs. Cost reduction and security concerns are significant factors, affecting the relative advantage positively, and negatively, respectively. Relative advantage also negatively affects complexity. Competitive pressure, technological readiness, and compatibility positively influence top management support. 
Government should tend to enhance the IT infrastructure of the country and balance trust, transparency, and neutrality among the actors, including operators, cloud providers, and SMEs in Turkey. Operators should also invest more in fiber infrastructure to enable service providers to provide mature cloud services in Turkey. In addition, the government should remove the obstacles hindering international cloud providers from entering the Turkish market where there is a lack of regulation that the data is not under a safe harbor agreement in Turkey. Moreover, SMEs should train their IT staff not only for implementation but also for integration and migration to grow in-house consultants, and experienced IT staff. For IaaS, well-known cloud providers are in the Turkish market that SMEs' service level agreement contracts are fully dependent on cloud providers, which may increase the cost. For PaaS and SaaS that have more options, cloud providers should invest more for mature cloud services in Turkey. Even there are $\mathrm{PaaS}$ and SaaS services in the market, the government should acknowledge SMEs to increase the awareness of CC and give incentives for trials of cloud services. Internet bandwidth, broadband subscriptions, and broadband data connection speeds should be increased for the benefits of these cloud services to attract cloud providers so that SMEs will have options to select reasonable cloud-based services, minimizing the perceived risks and cost. However, there are barriers to using those services from multiple cloud providers, because the integration costs of the distinct providers' services can be high, and a high level of IT staff or in-house consultants is required.

Future research is required to examine the sectoral division of SMEs such as manufacturing, and service sector or public and private sector, and will be extended by measuring Trialability within DOI theory, and Regulatory support within TOE theory. Product as a service segmentation will be able to be divided for these distinct sectors such as hardware, servers in terms of IaaS, software platforms, operating system in terms of PaaS, and CRM, supply chain systems in terms of SaaS.

\section{7- Declarations}

\section{7-1-Author Contributions}

C.S. collected the company data and developed the model and the conceptual framework; T.E. developed a draft plan and formed a summary together with C.S; C.S. and T.E. and contribution to the cloud computing literature review. All authors have read and agreed to the published version of the manuscript.

\section{7-2-Data Availability Statement}

The data presented in this study are available in article.

\section{7-3- Funding}

The authors received no financial support for the research, authorship, and/or publication of this article.

\section{7-4-Acknowledgements}

We would like to thank survey respondents in Izmir in Turkey for their time and participation.

\section{7-5- Conflicts of Interest}

The authors declare that there is no conflict of interests regarding the publication of this manuscript. In addition, the ethical issues, including plagiarism, informed consent, misconduct, data fabrication and/or falsification, double publication and/or submission, and redundancies have been completely observed by the authors.

\section{8- References}

[1] Santinha, G., and Antonio Soares. "SMEs and ICTs Adoption: A New Challenge to Regional Policies." 44th Congress of the European Regional Science Association: Regions and Fiscal Federalism European Regional Science Association (ERSA). Available online: https://www.econstor.eu/bitstream/10419/117159/1/ERSA2004_435.pdf (accessed on August 2021).

[2] Yildirim, F. "How Marketing and Customers Will Be Affected By Cloud Computing?” International Journal of eBusiness and eGovernment Studies 4, no. 2 (2012): 27-35.

[3] Singh, Shweta, and Arun Kumar Tripathi. “Analyzing for Performanc Factors in Cloud Computing.” International Journal of Control Theory and Applications 9, no. 21 (2016): 87-96.

[4] Şener, Sefer, Mesut Savrul, and Orhan Aydın. "Structure of Small and Medium-Sized Enterprises in Turkey and Global Competitiveness Strategies.” Procedia - Social and Behavioral Sciences 150 (2014): 212-21. doi:10.1016/j.sbspro.2014.09.119.

[5] AKTEPE, Çağlar, and Ömür Yaşar Saatçioğlu "Cloud Computing Adoption in Logistics Firms in Turkey: An Exploratory Study.” Sosyal Bilimler Arastirmalari Dergisi 7, no. 1 (2017): 9-20.

[6] Thakur, Neeti, Dhananjay Bisen, Vikas Rohit, and Neelesh Gupta. "Review on Cloud Computing: Issues, Services and Models." International Journal of Computer Applications 91, no. 9 (2014): 34-39. doi:10.5120/15912-5120. 
[7] Bradford, Marianne. "Modern ERP: Select, Implement \& Use Today’s Advanced Business Systems.” ISBN 9781 (2015): 284.

[8] Priyadarshinee, Pragati, Manoj Kumar Jha, Rakesh D. Raut, and Manoj G. Kharat. "Risk Analysis in Adoption of Cloud Computing in SMEs - A Literature Review.” International Journal of Business Information Systems 23, no. 1 (2016): 54-86. doi:10.1504/IJBIS.2016.078023.

[9] Keskin, N, A N Kiran, F K Egdemir, and T Eren. "Cloud Computer Service Provider Selection with Multicriteria Decision Making Methods.” International Journal of Management Information Systems and Computer Science, n.d., 52-73.

[10] Neicu, Andra Ileana, Anamaria Catalina Radu, Gheorghe Zaman, Ivona Stoica, and Florian Rapan. "Cloud Computing Usage in SMEs. An Empirical Study Based on SMEs Employees Perceptions.” Sustainability (Switzerland) 12, no. 12 (2020). doi:10.3390/su12124960.

[11] Nagahawatta, Ruwan, Matthew Warren, Sachithra Lokuge, and Scott Salzman. "Security Concerns Influencing the Adoption of Cloud Computing by SMEs: A Literature Review." In 27th Annual Americas Conference on Information Systems, AMCIS (2021).

[12] McKinsey Global Institute. The Age of Analytics: Competing in a Data-driven World. (2016). Available online: https://mck.co/3H8xYLN (accessed on August 2021).

[13] Cisco. Cisco Global Cloud Index (2015-2020). Available online: https://bit.ly/3eE6113 (accessed on August 2021).

[14] Cisco. Cisco Annual Internet Report (2018-2023). Available online: https://bit.ly/3my0oXI (accessed on August 2021).

[15] OC \& C Strategy Consultants. Tech Entrepreneurship Ecosystem in Turkey (2018). Available online: https://www.occstrategy.com/media/1298/tech-entrepreneurship-ecosystem-in-turkey.pdf (accessed on August 2021).

[16] Caputo, A. C., F. Cucchiella, L. Fratocchi, P. M. Pelagagge, and F. Scacchia. "A Methodological Framework for Innovation Transfer to SMEs.” Industrial Management and Data Systems 102, no. 5 (2002): 271-83. doi:10.1108/02635570210428302.

[17] Deloitte. Information and Communication Technologies (ICT) 2019 Market Data. (2020). Available online: http://www.tubisad.org.tr/en/images/pdf/tubisad_ict_2020.pdf (accessed on July 2021).

[18] Rogers, Everett M. “Diffusion of Innovations. n. p.: 3rd Edition” (1983). Macmillan Publishing. ISBN 9780029266502.

[19] Tornatzky, Louis G., Mitchell Fleischer, and Alok K. Chakrabarti. "Processes of technological innovation." Lexington Books, (1990).

[20] Davis, Fred D. "Perceived Usefulness, Perceived Ease of Use, and User Acceptance of Information Technology." MIS Quarterly: Management Information Systems 13, no. 3 (1989): 319-39. doi:10.2307/249008.

[21] Fishbein, Martin, and Icek Ajzen. "Belief, attitude, intention, and behavior: An introduction to theory and research." Philosophy and Rhetoric 10, no. 2 (1977).

[22] Rogers, Everett M. "Diffusion of Innovations: Modifications of a Model for Telecommunications." In Die Diffusion von Innovationen in Der Telekommunikation, 25-38, 1995. doi:10.1007/978-3-642-79868-9_2.

[23] Oliveira, Tiago, Manoj Thomas, and Mariana Espadanal. "Assessing the Determinants of Cloud Computing Adoption: An Analysis of the Manufacturing and Services Sectors." Information and Management 51, no. 5 (2014): 497-510. doi:10.1016/j.im.2014.03.006.

[24] Mundfrom, Daniel J., Dale G. Shaw, and Tian Lu Ke. "Minimum Sample Size Recommendations for Conducting Factor Analyses.” International Journal of Testing 5, no. 2 (2005): 159-68. doi:10.1207/s15327574ijt0502_4.

[25] Tornatzky, Louis G., and Katherine J. Klein. "Innovation Characteristics and Innovation Adoption-Implementation: A MetaAnalysis of Findings." IEEE Transactions on Engineering Management EM-29, no. 1 (1982): 28-45. doi:10.1109/TEM.1982.6447463.

[26] Arifin, W. N. “Confirmatory Factor Analysis and Raykov's rho. dim (data. cfa).” (2019). Available online: https://wnarifin.github.io/workshop/qvw2019/cfa.pdf (accessed on August 2021).

[27] Hair, Joseph F. Jr., William C. Black, Berry J. Babin and Rolph E. Anderson. "Multivariate Data Analysis. n.p.: Pearson, 7th edition" (2010). ISBN 9780138132637.

[28] Hu, Li Tze, and Peter M. Bentler. "Fit Indices in Covariance Structure Modeling: Sensitivity to Underparameterized Model Misspecification.” Psychological Methods 3, no. 4 (1998): 424-53. doi:10.1037/1082-989X.3.4.424.

[29] Sarstedt, Marko, Christian M. Ringle, and Joseph F. Hair. "Partial Least Squares Structural Equation Modeling." Handbook of Market Research (2017): 1-40. doi:10.1007/978-3-319-05542-8_15-1.

[30] Hair Jr., Joseph F., G. Tomas M. Hult, Christian M. Ringle, Marko Sarstedt, Julen Castillo Apraiz, Gabriel A. Cepeda Carrión, José Luis Roldán, et al. "Manual de Partial Least Squares Structural Equation Modeling (PLS-SEM) (Segunda Edición)" (July 9, 2019). doi:10.3926/oss.37. 
[31] Faynberg, Igor, Hui-Lan Lu, and Dor Skuler. Cloud Computing - Business Trends and Technologies. Vol. 41. Ltd: John Wiley \& Sons, 2016. doi:10.1002/9781118736074.

[32] Al Ajmi, Qasim, Ruzaini Abdullah Arshah, Adzhar Kamaludin, Ali Safa Sadiq, and Mohammed A. Al-Sharafi. "A Conceptual Model of e-Learning Based on Cloud Computing Adoption in Higher Education Institutions." 2017 International Conference on Electrical and Computing Technologies and Applications (ICECTA) (November 2017). doi:10.1109/icecta.2017.8252013.

[33] Bhuyan, Sambit, and Manoranjan Dash. "Exploring Cloud Computing Adoption in Private Hospitals in India: An Investigation of DOI and TOE Model." Journal of Advanced Research in Dynamical and Control Systems 10, no. 8 Special Issue (2018): $443-51$.

[34] Lynn, Theo, Xiaoning Liang, Anna Gourinovitch, John Morrison, Grace Fox, and Pierangelo Rosati. "Understanding the Determinants of Cloud Computing Adoption for High Performance Computing." Proceedings of the 51st Hawaii International Conference on System Sciences (2018). doi:10.24251/hicss.2018.489.

[35] Alismaili, Salim, Mengxiang Li, Jun Shen, and Qiang He. "A Multi Perspective Approach for Understanding the Determinants of Cloud Computing Adoption among Australian SMEs.” In ACIS 2015 Proceedings - 26th Australasian Conference on Information Systems, (2015).

[36] Janssen, Marijn, and Anton Joha. "Challenges for Adopting Cloud-Based Software as a Service (SAAS) in the Public Sector." In 19th European Conference on Information Systems, ECIS 2011, 2011. http://aisel.aisnet.org/ecis2011/80.

[37] Charlebois, Kathleen, Nicole Palmour, and Bartha Maria Knoppers. "The Adoption of Cloud Computing in the Field of Genomics Research: The Influence of Ethical and Legal Issues." PLoS ONE 11, no. 10 (2016): 1-33. doi:10.1371/journal.pone.0164347.

[38] Sayginer, C., and Tuncay Ercan. "Cloud Computing Adoption by National Sector in Turkey: A Framework based on DOI Theory.” Proceedings Book of V. International European Congress on Social Sciences. (2020). ISBN 9786257898157.

[39] Alshamaila, Yazn, Savvas Papagiannidis, and Feng Li. "Cloud Computing Adoption by SMEs in the North East of England: A Multi-Perspective Framework." Journal of Enterprise Information Management 26, no. 3 (2013): $250-75$. doi:10.1108/17410391311325225.

[40] Hasimi Sallehudin, Razli Che Razak, and Mohammad Ismail. "Factors Influencing Cloud Computing Adoption in the Public Sector: An Empirical Analysis.” Journal of Entrepreneurship and Business 3, no. 1 (2015): 30-45. doi:10.17687/jeb.0301.03.

[41] Hassan, H., and H. M. Nasir. "Determinants of Cloud Computing Adoption at Firm Level: From the Technological Context." Journal of Engineering and Applied Sciences 12, no. 16 (2017): 4186-92. doi:10.3923/jeasci.2017.4186.4192.

[42] Mas'adeh, Ra'ed. "Cloud Computing Perceived Importance in the Middle Eastern Firms: The Cases of Jordan, Saudi Arabia and United Arab Emirates from the Operational Level." Communications and Network 08, no. 03 (2016): $103-117$. doi:10.4236/cn.2016.83011.

[43] Klug, W, and X Bai. "Factors Affecting Cloud Computing Adoption among Universities and Colleges in the United States and Canada.” Issues In Information Systems 16, no. 111 (2015): 1-10. doi:10.48009/3_iis_2015_1-10.

[44] Senyo, Prince Kwame, John Effah, and Erasmus Addae. "Preliminary Insight into Cloud Computing Adoption in a Developing Country.” Journal of Enterprise Information Management 29, no. 4 (2016): 505-24. doi:10.1108/JEIM-09-2014-0094.

[45] Gangwar, Hemlata, Hema Date, and R. Ramaswamy. "Understanding Determinants of Cloud Computing Adoption Using an Integrated TAM-TOE Model.” Journal of Enterprise Information Management 28, no. 1 (2015): 107-130. doi:10.1108/JEIM08-2013-0065.

[46] Gutierrez, Anabel, Elias Boukrami, and Ranald Lumsden. "Technological, Organisational and Environmental Factors Influencing Managers' Decision to Adopt Cloud Computing in the UK." Journal of Enterprise Information Management 28, no. 6 (2015): 788-807. doi:10.1108/JEIM-01-2015-0001.

[47] Alkhalil, Adel, Reza Sahandi, and David John. "An Exploration of the Determinants for Decision to Migrate Existing Resources to Cloud Computing Using an Integrated TOE-DOI Model.” Journal of Cloud Computing 6, no. 1 (2017). doi:10.1186/s13677-016-0072-x.

[48] Deil, Robert, and Philipp Brune. "Cloudy with a Chance of Usage? - Towards a Model of Cloud Computing Adoption in German SME.” In CEUR Workshop Proceedings, edited by X Franch, J Ralyté, R Matulevičius, C Salinesi, and R Wieringa, 1848:65-72, 2017.

[49] Kyriakou, Niki, Manolis Maragoudakis, Euripidis Loukis, and Marinos Themistocleous. "Prediction of Propensity for Enterprise Cloud Computing Adoption.” Proceedings of the 50th Hawaii International Conference on System Sciences (2017) (2017). doi:10.24251/hicss.2017.596.

[50] Al-Hujran, Omar, Enas M. Al-Lozi, Mutaz M. Al-Debei, and Mahmoud Maqableh. "Challenges of Cloud Computing Adoption from the TOE Framework Perspective." International Journal of E-Business Research 14, no. 3 (2018): 77-94. doi:10.4018/IJEBR.2018070105. 
[51] Pathan, Zulfiqar Hussain, Zeng Jianqiu, Umair Akram, Muhammad Kaleem Khan, Zahid Latif, and M. Zahid Tunio. "Innovation-Diffusion Determinants of Cloud-Computing Adoption by Pakistani SMEs." Human Systems Management 36, no. 3 (2017): 197-209. doi:10.3233/HSM-171794.

[52] Tashkandi, Al Alaa, and Ibrahim Al-Jabri. "Cloud Computing Adoption by Higher Education Institutions in Saudi Arabia: Analysis Based on TOE.” In 2015 International Conference on Cloud Computing, ICCC 2015. doi:10.1109/cloudcomp.2015.7149634.

[53] Guner, E. O., and Eriks Sneiders. "Cloud Computing Adoption Factors in Turkish Large-scale Enterprises.” PACIS 2014 Proceedings (2014). https://aisel.aisnet.org/pacis2014/353 (accessed on August 2021).

[54] Arpaci, Ibrahim. "Antecedents and Consequences of Cloud Computing Adoption in Education to Achieve Knowledge Management.” Computers in Human Behavior 70 (2017): 382-90. doi:10.1016/j.chb.2017.01.024.

[55] The Software Alliance. 2018 BSA Global Cloud Computing Scorecard- Powering a Bright Future. (2018). Available online: https://cloudscorecard.bsa.org/2018/pdf/BSA_2018_Global_Cloud_Scorecard.pdf (accessed on August 2021).

[56] ITA, International Trade Administration. Turkey - Information and Communication Technology. (2019). Available online: https://www.export.gov/apex/article2?id=Turkey-Information-and-Communications-Technology (accessed on August 2021).

[57] Oliveira, L.R.De, A JulioMurlick, G V Pereira, and R Vicentin. “Adoption Analysis of Cloud Computing Services.” African Journal of Business Management 7, no. 24 (2013): 2362-74. doi:10.5897/AJBM12.1333.

[58] Hassan, Haslinda, Mohd Herry Mohd Nasir, Norhaiza Khairudin, and Iskandar Adon. "Factors Influencing Cloud Computing Adoption in Small and Medium Enterprises.” Journal of Information and Communication Technology 16, no. 1 (2017): $21-41$. doi:10.32890/jict2017.16.1.8216.

[59] Au-Yeung, B., D Chu, M Enfante, G Logan, and K Saelee. Industry Analysis: Cloud Computing. (2017). Available online: https://www.csus.edu/indiv/h/hattonl/documents/cloudcomputingindustry.pdf (accessed on August 2021). 